Original Article

\title{
Comparison of the Efficacy of Different Long-term Interventions on Chronic Low Back Pain Using the Cross-sectional Area of the Multifidus Muscle and the Thickness of the Transversus Abdominis Muscle as Evaluation Indicators
}

Qiuchen Huang, $\mathrm{PT}^{1-3)^{*}}$, Desheng Li, $\mathrm{PT}^{1-3)}$, Jing Zhang ${ }^{4)}$, Degang Yang $^{2,3)}$, Ming Huo, PT, PhD ${ }^{5)}$, Hitoshi Maruyama, PT, PhD $^{1)}$

1) Department of Physical Therapy, International University of Health and Welfare: 2600-1 Kitakanemaru, Ohtawara, Tochigi 324-8501, Japan

2) Department of Physical Therapy, China Rehabilitation Center, China

3) School of Rehabilitation Medicine, Capital Medical University, China

4) Xiluoyuan Community Health Service Center, China

5) Himeji Dokkyo University, Japan

\begin{abstract}
Purpose] The purpose of this study was to examine the different effects of long-term intervention between proprioceptive neuromuscular facilitation (PNF) and neuromuscular joint facilitation (NJF) patterns for the pelvis on chronic low back pain as assessed by the cross-sectional area of the multifidus muscle and the thickness of the transversus abdominis muscle. [Subjects] The subjects were 12 young people (five males, seven females) who had experienced chronic low back pain on one side for more than 6 months. [Methods] The subjects were treated by resting, PNF or NJF therapy, and each treatment was administered for one month. Ultrasonography was used to measure the changes in the transversus abdominis muscle thickness and the multifidus muscle cross-sectional area. [Results] The thickness of the transversus abdominis muscle and the cross-sectional area of the multifidus muscle in the NJF group, after resting, increased significantly and were higher than those in the PNF group. [Conclusion] The results show that significantly better improvement can be obtained for chronic low back pain by applying long-term intervention of NJF patterns.

Key words: Neuromuscular joint facilitation (NJF), Long-term intervention, Low back pain
\end{abstract}

(This article was submitted Apr. 3, 2014, and was accepted May 22, 2014)

\section{INTRODUCTION}

Chronic low back pain (LBP) is considered a recurring human disease. In order to walk upright and keep that position, humans exert a heavy burden on the lumbar region. Since many factors contribute to LBP (multifactorial disease), a simple treatment is not enough to manage $\mathrm{it}^{1)}$.

Chronic low back pain is defined as back pain lasting for more than 12 weeks, and it affects more than $50 \%$ of the general population. It is estimated that over $70 \%$ of adults have at least one episode of low back pain during their lifetimes. The prevalence of LBP is higher in young, economically active adults. Indeed, low back pain is the second most common reason for absenteeism, and one of the most common reasons for medical consultation ${ }^{2}$.

*Corresponding author. Qiuchen Huang (E-mail: qiuchen_1984@126.com)

(C2014 The Society of Physical Therapy Science. Published by IPEC Inc. This is an open-access article distributed under the terms of the Creative Commons Attribution Non-Commercial No Derivatives (by-ncnd) License $<$ http://creativecommons.org/licenses/by-nc-nd/3.0/>.
The muscles of the trunk, which are involved in maintaining stability, can be classified into the global muscle system and the local muscle system. Trunk movement is controlled by the global muscle system, which supports the spine and consists shallow lumbar muscles.

The local muscle system contains many muscles, such as the multifidus, longissimus lumborum, iliocostalis lumborum, intertransversalis, and interspinal muscles, medial fiber of the lumbar quadrate muscle. The internal abdominal oblique muscle (the fibers adhering to the fascia thoracolumbalis) is a constituent of the local muscle system.

The roles in the stabilization of the lumbar region of the multifidus and transversus abdominis muscles have been reported. The activities of the inner muscles (multifidus and transverse abdominis muscles) are highly correlated with the stability of the lumbar region ${ }^{3)}$.

An important risk factor of low back pain is its weakness and its lack of motor control in the local muscle system, which contains the lumbar multifidus and transversus abdominis muscles ${ }^{4}$. In the case of low activity of inner muscles, the global muscle system (erector spinae, rectus abdominis, and abdominal oblique muscles) compensate 
to maintain the stabilization of the lumbar region, and this compensation is one of the causes of $\mathrm{LBP}^{5}$ ).

Intra-abdominal pressure is increased by the tonus of the fascia thoracolumbalis, and this is affected by contraction of the transversus abdominis and multifidus muscles. The increase in fascia thoracolumbalis tonus and the intraabdominal pressure resulting from muscle contraction also contributes to the regional support of each spinal vertebra. In particular, support of the sacroiliac joint, one of the structures which is essential for spinal stabilization, is affected by the transversus abdominis muscle and the lower fibers of the internal abdominal oblique muscle ${ }^{6}$.

When humans engage in some activities, contraction of the transversus abdominis muscle occurs ahead of the trunk muscle groups ${ }^{7}$, and in preparation for trunk movement, the intra-abdominal pressure (IAP) would increases.

Currently, rapid progress is being made in research on the pelvic floor muscles. The stabilization of the lumbar region is determined by the inner unit, which is composed of pelvic floor muscles, and the transversus abdominis and multifidus muscles, and diaphragm. Active approaches to the treatment of urinary incontinence and low back pain have been put into clinical practice.

In clinical research, the resistance movement of the pelvis, the pattern of proprioceptive neuromuscular facilitation (PNF), neuromuscular joint facilitation (NJF), and mobilization are always used in the treatment of LBP, and static contraction is selected in clinical treatment. However, this treatment only relieves symptoms temporarily, and its effect does not last long.

NJF is a new therapeutic exercise based on kinesiology which integrates the facilitation element of proprioceptive neuromuscular facilitation and joint composition movement, aiming to improve the movement of the joint through passive exercise, active exercise, and resistance exercise ${ }^{8)}$. It is used to increase the strength, flexibility, and range of motion $(\mathrm{ROM})^{9}$ ). Conservative therapy is also used in the treatment of more than $90 \%$ of patients with low back pain, and "postural exercise" has been widely used in clinical treatments for the prevention of lumbar lordosis and strengthening of the multifidus muscle. However, because there is no objective evaluation, its efficacy is controversial.

Ultrasound imaging has been advocated as a noninvasive method for quantifying muscle morphology and behavior, and it has been increasingly used both in research and as a clinical tool throughout the rehabilitation process.

Ultrasound imaging has been validated as a measure of lumbar multifidus muscle morphology, through comparison with magnetic resonance imaging measurements, and as an indicator of muscle activation with indwelling electromyography $^{10)}$.

The immediate effects of different treatments of LBP have been assessed using the cross-sectional area of the multifidus muscle measured by ultrasound imaging. However, there has been no research about the efficacy of different long-term interventions ${ }^{11)}$.

The aim of this study was to examine the different effects of long-term interventions using the PNF and NJF patterns on the pelvis.
Table 1. Subject characteristics

\begin{tabular}{lc}
\hline & $\mathrm{M} \pm \mathrm{SD} \mathrm{N}=12$ \\
\hline Age $(\mathrm{y})$ & $25.9 \pm 5.3$ \\
Height $(\mathrm{cm})$ & $174.0 \pm 9.4$ \\
Weight $(\mathrm{kg})$ & $70.3 \pm 17.6$ \\
VAS & $3.8 \pm 2.8$ \\
\hline
\end{tabular}

\section{SUBJECTS AND METHODS}

The subjects were twelve young people ( 5 males, 7 females) who had suffered chronic low back pain for more than 6 months on one side of their bodies. Subject characteristics are detailed in Table 1. The purpose and contents of this research were explained to the subjects, and all subjects gave their informed consent to participation in this study. The study was approved by the Research Ethics Committee of the International University of Health and Welfare, and the IRD number for this study is $12-155$.

Before the treatment, the subjects were evaluated for pain severity using a visual analogue scale (VAS), and the cross-sectional area of the multifidus muscle and the thickness of the transversus abdominis muscle of the subjects were measured using ultrasound imaging.

The subjects were joined the intervention group and control group. For the control group, all subjects rested for one month. For the intervention group, the subjects were divided into PNF and NJF groups, two interventions were carried out: the front inferior pelvic pattern of PNF, and the front inferior pelvic pattern of NJF. In the PNF group, the front inferior pelvic pattern of PNF was carried out 3 times every week. In the NJF group, the front inferior pelvic of NJF was carried out 3 times every week.

For the front inferior pelvic pattern of PNF, the examiner placed his hands on the subjects' knees and applied traction and resistance while they performed the front inferior pelvic pattern. The static resistance and the traction were applied in the intermediate position of the PNF pattern by the examiner.

For the front inferior pelvic pattern of NJF, one hand of the examiner was placed against the knee, and traction and resistance were applied as in the PNF pattern. The other hand of the examiner was placed on the spinous process of L3 to prevent upward curvature. All interventions were carried out by one physiotherapist.

In both the PNF and NJF interventions, the maximum contraction time of 5 seconds was maintained for the intermediate position in both patterns used for the interventions.

Before, and after one month of PNF and NJF treatments, the VAS scores, cross-sectional areas of the multifidus muscle and thicknesses of the transversus abdominis muscle were measured using ultrasound imaging. Each measurement was carried out twice, and the average value was used for analysis. Ultrasound images of the multifidus muscle wall and transversus abdominis were obtained using a SonoSite ultrasound system (SonoSite180 PLUS, B mode, $5 \mathrm{MHz}$ linear transducer). Gel was interposed between the transducer and the skin. In order to measure the thickness 
Table 2. VAS and thickness of the transversus abdominis muscle (cm)

\begin{tabular}{lcllll}
\hline & \multicolumn{2}{c}{$\begin{array}{c}\text { Thickness of the transversus } \\
\text { abdominis muscle }(\mathrm{cm})\end{array}$} & \multicolumn{2}{c}{ VAS } \\
& \multicolumn{2}{c}{$\mathrm{a}<\mathrm{d}^{* *}$} & $3.8 \pm 2.8$ & \\
a.Before treatment & $0.46 \pm 0.04$ & & $4.0 \pm 2.5$ & \\
b.Resting & $0.42 \pm 0.05$ & $\mathrm{~b}<\mathrm{c} . \mathrm{d}^{*}$ & $3.1 \pm 2.3$ & $\mathrm{c}<\mathrm{a} . \mathrm{b}^{*}$ \\
c.PNF & $0.48 \pm 0.05$ & $\mathrm{c}<\mathrm{d}^{*}$ & & $2.3 \pm 2.5$ & $\mathrm{~d}<\mathrm{a} . \mathrm{b} . \mathrm{c}^{* *}$ \\
d.NJF & $0.60 \pm 0.06$ & & &
\end{tabular}

Table 3. Cross-sectional area of the multifidus $\left(\mathrm{cm}^{2}\right)$

\begin{tabular}{lcccc}
\hline & \multicolumn{2}{c}{ Pain side } & \multicolumn{2}{c}{ No pain side } \\
\hline a.Beore treatment & $7.7 \pm 1.8$ & $\mathrm{a}<\mathrm{c} . \mathrm{d}^{* *}$ & $8.5 \pm 2.2$ & $\mathrm{a}<\mathrm{d}^{*}$ \\
b.Rest & $7.0 \pm 1.7$ & $\mathrm{~b}<\mathrm{a} . \mathrm{c} . \mathrm{d}^{* *}$ & $8.6 \pm 2.3$ & $\mathrm{~b}<\mathrm{d}^{*}$ \\
c.PNF & $7.9 \pm 2.3$ & $\mathrm{c}<\mathrm{d}^{* *}$ & $8.8 \pm 1.9$ & $\mathrm{c}<\mathrm{d}^{*}$ \\
d.NJF & $8.7 \pm 2.0$ & & $9.5 \pm 2.1$ & \\
\hline$* \mathrm{p}<0.05 * * \mathrm{p}<0.01$ & & &
\end{tabular}

of the transversus abdominis muscle, the transducer was positioned adjacent to and perpendicular to the abdominal wall, $25 \mathrm{~mm}$ anteromedial to the midpoint between the ribs and ilium on the midaxillary line, and parallel to the muscle fibers of the transversus abdominis ${ }^{12}$. For the measurement of the cross-sectional area of the multifidus muscle, the transducer was placed on the skin $25 \mathrm{~mm}$ distal from the spinous process of L3 and vertical to the vertebral column.

To avoid inter-rater errors, the same physical therapist performed all the measurements. Ultrasound images were saved as still images, and the thickness and cross-sectional area measurements were performed for muscle only, that is, between the fascia boundaries.

In order to determine the minimum effect of the 2 intervention methods, one-way analysis of variance with the Bonferroni correction was used. The factors were the VAS score, the thickness of the transversus abdominis muscle, and the cross-sectional area of the multifidus muscle. The data were analyzed using SPSS Ver. \#17.0 for Windows.

\section{RESULTS}

The results for VAS and thickness of the musculus transversus abdominis the 12 subjects are shown in Table 2.

The VAS score decreased significantly in the PNF and NJF groups compared with before treatment and the rest of the group. The decrease in the NJF group was the most significant.

The thickness of the transversus abdominis muscle increased significantly in the PNF and NJF groups compared with the pre-treatment value and the rest group value, and the increase in the NJF group was the most significant.

The results for the cross-sectional area of the multifidus muscle are shown in Table 3. The cross-sectional area of the multifidus muscle on the side with pain of the PNF, and NJF groups showed significant increases compared to the pre-treatment value and the rest group value. The increase of the NJF group was the greatest. On the side without pain, the cross-sectional areas of the multifidus muscle of the NJF groups showed significant increases.

\section{DISCUSSION}

It is generally recognized that the multifidus muscle and transversus abdominis muscle are very important for the stabilization of the trunk. However, in clinical research, the contribution of the transversus abdominis has been comparatively ignored.

Traditional remedies such as traction mobilization show immediate effects, but only a few studies have investigated their long-term treatment effects. Recently, special emphasis has been placed on combination therapies comprising LBP exercise and lumbar stabilization exercise, but no objective assessment of traditional methods has been performed.

In this study, the effect of LBP treatment was evaluated by measuring the thickness of the transversus abdominis muscle, and the cross-sectional area of the multifidus muscle using ultrasound imaging. The PNF and NJF techniques elicited different effects. Transverse abdominis muscle thickness and the cross-sectional area of the multifidus muscle on the side with pain increased significantly after applying the PNF technique, In the NJF pattern, proximal resistance is exerted, promoting the isometric contraction of the multifidus muscle, and the transverse abdominis muscle also would be treated together with multifidus muscle contraction and increased intra-abdominal pressure.

The combination of multifidus muscle and transverse abdomen is muscle treatment by NJF promoted strengthening of the local muscle system, and our results suggest that imbalance of the multifidus muscle and weakness of the transverse abdomenis muscle can be effectively improved by long-term treatment with the NJF pattern.

Future studies are still needed to investigate the persistence of treatment effects of different treatments after longterm interventions for patients with low back pain. 
1854 J. Phys. Ther. Sci. Vol. 26, No. 12, 2014

\section{REFERENCES}

1) Kai S: Consideration of low back pain in health and welfare workers. J Phys Ther Sci, 2001, 13: 149-152. [CrossRef]

2) Inoue $Y$, Nagakura $H$, Uesugi $M$, et al.: The influence of low back pain on care workers' health-related quality of life. J Phys Ther Sci, 2011, 23 603-606. [CrossRef]

3) Hides JA, Jull GA, Richardson CA: Long-term effects of specific stabilizing exercises for first-episode low back pain. Spine, 2001, 26: E243-E248. [Medline] [CrossRef]

4) Hides JA, Richardson CA, Jull GA: Multifidus muscle recovery is not automatic after resolution of acute, first-episode low back pain. Spine, 1996 , 21: 2763-2769. [Medline] [CrossRef]

5) Han IK, Kim SY, Kim TY: Comparison of changes in abdominal muscle thickness using ultrasound imaging during the abdominal drawing-in maneuver performed by patients with low back pain and healthy subjects. J Phys Ther Sci, 2012, 24: 383-385. [CrossRef]

6) McGill SM: A revised anatomical model of the abdominal musculature for torso flexion efforts. J Biomech, 1996, 29: 973-977. [Medline] [CrossRef] 7) Richardson C: Spinal Segmental Stability. In: Therapeutic Exercise for Spinal Segmental Stabiliationin Low Back Pain, Edinburgh: Churchill Livingstone, 1999, pp 16-26.

8) Huo M: Neuromuscular Joint Facilitation. Tokyo: IPEC press, 2010, p 3.

9) Wang H, Huo M, Huang Q, et al.: The immediate effect of neuromuscula joint facilitation (NJF) treatment on hip muscle strength. J Phys Ther Sci, 2013, 25: 1455-1457. [Medline] [CrossRef]

10) Hides J, Wilson S, Stanton W, et al.: An MRI investigation into the function of the transversus abdominis muscle during "drawing-in" of the abdominal wall. Spine, 2006, 31: E175-E178. [Medline] [CrossRef]

11) Huang Q, Li D, Yokotsuka N, et al.: The intervention effects of different treatment for chronic low back pain as assessed by the cross-sectional area of the multifidus muscle. J Phys Ther Sci, 2013, 25: 811-813. [Medline] [CrossRef]

12) Critchley D: Instructing pelvic floor contraction facilitates transversus abdominis thickness increase during low-abdominal hollowing. Physiother Res Int, 2002, 7: 65-75. [Medline] [CrossRef] 